\title{
Photochemical crosslinking of collagen and Poly (vinyl pyrrolidone) hydrogel system for biomedical applications by using novel photo initiators
}

\author{
B. Chandrika Ambati, Sinead M. Devery, Clement L. Higginbotham \\ Centre for Biopolymer and Biomolecular Research, Athlone Institute of Technology, \\ Dublin Road, Athlone, Co Westmeath, Ireland.
}

\begin{abstract}
Photochemical crosslinking is a best alternative method when compared to conventional crosslinking methods such as chemical and physical methods, by using vitamins like riboflavin and ascorbic acid as photochemical initiators to enhance the physicochemical properties of collagen poly (vinyl pyrrolidone) blend without any toxic by products. Collagen and poly (vinyl pyrrolidone) blends were investigated by Fourier transform infrared spectroscopy (FTIR), Differential scanning calorimetry (DSC), Swelling studies, Tensile testing and the surface properties were studied by Contact angle measurements and Microscopy. Collagen and PVP blended samples were irradiated with the UV light wavelength $\lambda=300 \mathrm{~nm}$ for 30 minutes by using $0.03 \%$ riboflavin and ascorbic acid as photosensitizers and the samples were air dried at room temperature. CollagenPVP blended hydrogels showed good swelling properties and the lower contact angles indicate their hydrophilic nature. The photochemical crosslinking also improved the thermal stability of the hydrogels which can be confirmed by broad endothermic peak between $80-180^{\circ} \mathrm{C}$ in the thermoscan. The FTIR spectral changes clearly exhibited that these two polymers were strongly crosslinked and miscible.
\end{abstract}

Keywords - Collagen, Poly (vinyl pyrrolidone), Photochemical crosslinking, Vitamins, Hydrogel, Drug delivery

\section{Introduction}

Collagen is the most abundant and ubiquitous protein in our body [1] and is the major component in the skin, bones and cartilage. It is treated as a good biomaterial for drug delivery and tissue engineering [2] from decades because of its excellent biocompatibility [3] and biodegradation properties. However applications of collagen are limited because of its weak mechanical properties, degradation upon swelling and low processability and intolerance to chemical and enzymatic attacks in the body [4]. To enhance the applications of collagen as a drug delivery vehicle and as a scaffold in tissue engineering, there is a need for modification methods to improve its physicochemical properties without compromising stability and biocompatibility [5]. Poly (vinyl pyrrolidone) is a water soluble synthetic polymer with good absorption and complex forming properties [6]. The hydrophobic methylene group and hydrophilic amide group ratios in the polymer makes PVP as a one of the best choices in the biomedical field for preparation of synthetic plasma and as a carrier for drug delivery [7] [8] [9]. Polymer blending is an interesting criterion to create new materials for wide usage in drug delivery, wound dressing and as a scaffold for tissue engineering [10]. Collagen and Poly (vinyl pyrrolidone) based hydrogels are more interested due to their excellent biocompatibility and biodegradative features [11]. Collagen and PVP are completely miscible and interacts with each other by formation of inter and intra molecular hydrogen bonding [12]. This can be best achieved by photo polymerization which mainly involves exposure to light in presence of photo reactive species to generate free radicals which in turn helps in the formation of bonding in the hydrogels [13] [14].

The previous research supports the use of dyes like proflavin [15], methylene blue [16] and rose Bengal [17] as photochemical initiators for crosslinking of different biomaterials. Riboflavin [18] and ascorbic acid [19] particularly participate in hydroxylation of amino acids like hydroxyproline, proline and glycine [20] which in turn facilitates the crosslinking. In our laboratory we recently developed a non-toxic easy to use technique with the combination of UV light and the vitamins as photosensitizers to enhance the physicochemical properties such as tensile strength, swelling rate and thermostability of collagen based blends.

This study was mainly focused on proving that the riboflavin as well as ascorbic acid can acts as photo sensitizers, specifically it was aimed to study the use of non toxic photoinitiators to enhance photochemical crosslinking of collagen-PVP hydrogels. In this regard the principal hypothesis of this work was that photochemical crosslinking can generate hydrogels with greatest improved properties for protein delivery without any toxic crosslinking by-products. To examine this hypothesis the hydrogels were synthesized with ascorbic acid and riboflavin and studied their chemical and mechanical parameters to compare the effectiveness 
of these photo initiators as crosslinking agents. Followed by characterization, the gels were incorporated with selected model protein bovine serum albumin to understand it's release kinetics and ability to entrap the protein.

\section{Materials and Methods}

Collagen - Poly (vinyl pyrrolidone) blended hydrogels were prepared by modifying the previously described method by Makarand et al (2000) [21]. Calf bovine collagen type I was used as the source of collagen for the hydrogel preparation. All the other chemicals like PVP and buffer solutions were analytical grade and obtained from (Sigma, Germany). Riboflavin and ascorbic acid were purchased from (Sigma, Germany). 2\% collagen was dissolved by adding $0.25 \mathrm{mMol}$ acetic acid and stirred overnight with a magnetic stirrer (Sartorius, AGB Scientifics Ltd) at room temperature. The homogeneous paste was blended with PVP (4\%). To this mixture $0.03 \%$ of photo initiators were added and mixed thoroughly and then $0.5 \mathrm{ml}$ of collagen suspension was poured in a casting apparatus and kept under UV light for 30 minutes in an enclosed chamber. After the process of curing they were air dried and sealed in a vacuum pack for further use. In the same manner collagen and collagen - PVP blend was prepared without any photo initiator and used as a control samples.

\subsection{Surface morphology}

\section{Results and Discussion}

The surface morphology of hydrogels were determined using scanning probe light microscope (Olympus, BX60, Lab equip). Dried hydrogels were mounted on a glass slide and the images were scanned and recorded at the magnification of $50 \mathrm{x}$.

The surface morphology of hydrogels showed a good porosity with interconnected network like structures. There is a great change in surface morphology and strength in cross linked hydrogels when compared to non crosslinked control samples as shown in Fig. 1(a) and (d).

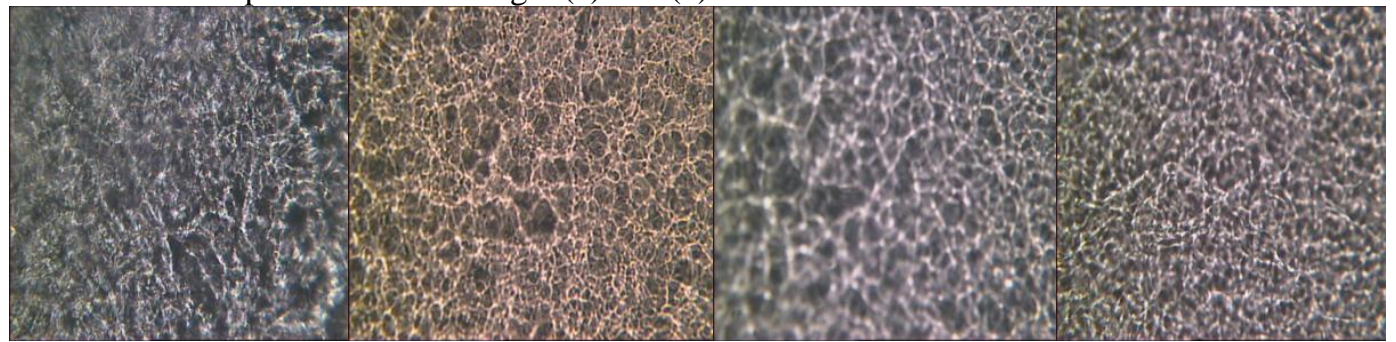

(a) (c) (d)

(a)Collagen, (b) Collagen -PVP with riboflavin, (c) Collagen -PVP with ascorbic acid,(d) Collagen-PVP

Figure. 1 Microscopic images of the hydrogels at 50X magnification

\subsection{Fourier transform infrared spectroscopy}

The FTIR spectra of the different treatment groups were shown in Fig. 2. IR spectra of these hydrogels are useful in predicting the secondary structure of the molecules and to elucidate inter and intra molecular interaction among the polymers. Non crosslinked collagen showed the amide A at around $3270 \mathrm{~cm}^{-1}$. The amide I and II were falling in 1627 and $1533 \mathrm{~cm}^{-1}$ respectively and amide III at $1259 \mathrm{~cm}^{-1}$. These positions are very sensitive in relation to change in conformation of the molecules and the water bonded to these molecules. In the present situation we observed a shift of amide A band towards higher wave numbers $\left(3280,3286 \mathrm{~cm}^{-1}\right)$ except in riboflavin $\left(2961 \mathrm{~cm}^{-1}\right)$ crosslinked hydrogels due to NH stretching coupled to hydrogen bonding and also a slight shift of amide I to lower wave number $\left(1625 \mathrm{~cm}^{-1}\right.$ and $\left.1624 \mathrm{~cm}^{-1}\right)$ and amide II and III shifting towards higher wave numbers (1539 and $\left.1545 \mathrm{~cm}^{-1}\right)$ when compared to non crosslinked collagen $\left(1533 \mathrm{~cm}^{-1}\right)$

Regarding the PVP blend without any crosslinking agent the amide A band position at $3286 \mathrm{~cm}^{-1}$, which was reduced to $3270 \mathrm{~cm}^{-1}$ and was diminished in riboflavin crosslinked blend and showed a band at $2962 \mathrm{~cm}^{-1}$ due to $-\mathrm{CH} 2$ asymmetrical stretching.

The amide I and amide II were also shifted towards lower wave numbers. There is no change in amide III band. This might be due to the complete mixing and uniformity of the collagen with PVP and the blend is homogenous. All these hydrogels exhibited the bands at around $3270-3286 \mathrm{~cm}^{-1}$ due to $\mathrm{NH}$ stretching coupled with hydrogen bonding and the bands between 2960 to $2962 \mathrm{~cm}^{-1}$ as a result of $-\mathrm{CH} 2$ asymmetric stretch and $\mathrm{C}=\mathrm{O}$ stretch or coupling of hydrogen bonding with $\mathrm{C}=\mathrm{O}$ at around $1650 \mathrm{~cm}^{-1}$ 


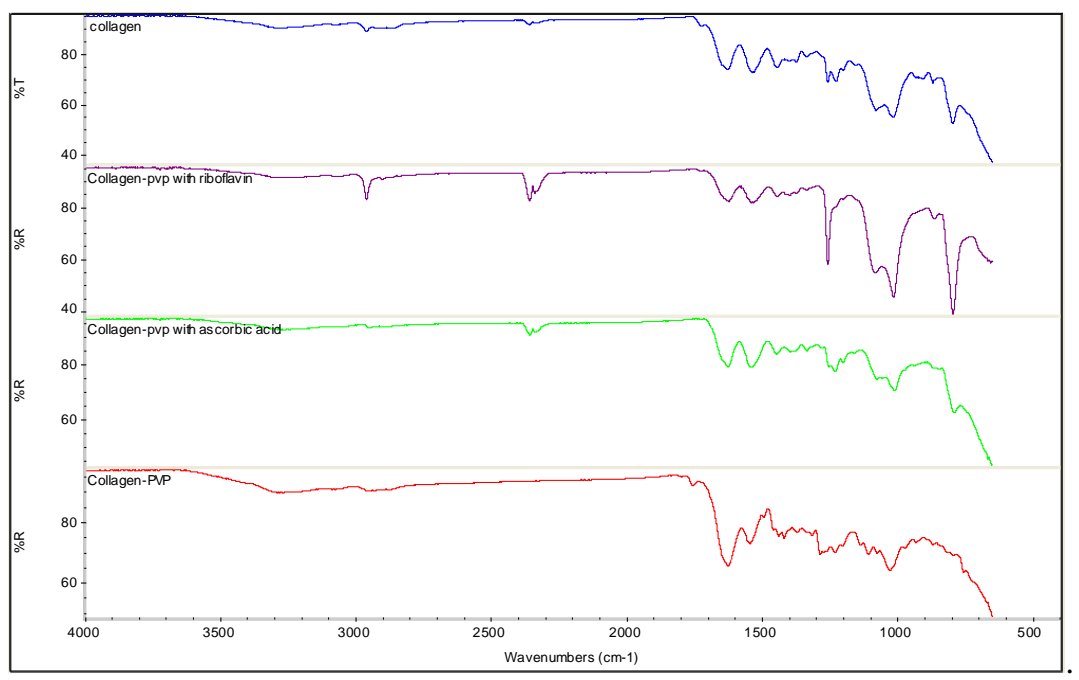

Figure. 2 FTIR spectra of different treatment groups of hydrogels

\subsection{Contact angle determination}

The contact angle between the liquid and solid surface of the polymer membrane determines the polar nature of the hydrogels. Hydrogel membranes were mounted on the space provided on the Goniometer (Olympus, TGHM, Germany) and $0.1-0.2 \mu \mathrm{l}$ of liquid drop was placed on the polymer membrane with the help of a micro syringe and the contact angles were measured with the two liquids water and glycerol. The values obtained for collagen and collagen -PVP blended samples are almost all similar indicating that these two polymers are well miscible and have good interaction with each other.

Table 1 Contact angles of hydrogels $\left(^{\circ}\right)$

\begin{tabular}{|l|l|l|l|l|}
\hline Liquid & Collagen & $\begin{array}{l}\text { Collagen-PVP with } \\
\text { riboflavin }\end{array}$ & $\begin{array}{l}\text { Collagen-PVP with } \\
\text { ascorbic acid }\end{array}$ & Collagen-PVP \\
\hline Water & 43 & 44 & 43 & 42 \\
\hline Glycerol & 59 & 62 & 65 & 66 \\
\hline
\end{tabular}

\subsection{Swelling studies}

To understand the swelling properties of these hydrogels, the dry weights were measured before immersing in the solution of phosphate-buffered saline (PBS) pH 7.0. At particular time intervals the hydrogels were removed from the solution and blotted with tissue paper to remove excess surface water and the wet weight of each hydrogel was measured with the help of a sensitive balance (Sartorius, AGB Scientifics Ltd). The same procedure was repeated at different time periods.

The following formula was used to calculate the swelling ratio of the hydrogels.

$$
\mathrm{Qs}=\frac{\mathrm{Ww}-\mathrm{Wd}}{\mathrm{Wd}}
$$

Where $\mathrm{Qs}=$ Swelling ratio

$\mathrm{Ww}=$ Wet weight and

$\mathrm{Wd}=$ Dry weight

The swelling patterns were followed as shown in Fig. 3 non crosslinked collagen exhibited rapid swelling with in $24 \mathrm{~h}$ time and reached to equilibrium. In case of crosslinked hydrogels there was a prolonged and regular rate of swelling until $120 \mathrm{~h}$ of time. After that they reached to equilibrium. Collagen-PVP blend without any crosslinking agent reached to maximum swelling at $24 \mathrm{~h}$ time and reached to equilibrium. In case of non crosslinked collagen the trend was similar to PVP blended hydrogels and they also showed the maximum swelling at $24 \mathrm{~h}$ of time. Basing on the swelling ratios we can conclude that the hydrogels were strongly crosslinked with increased stability. 


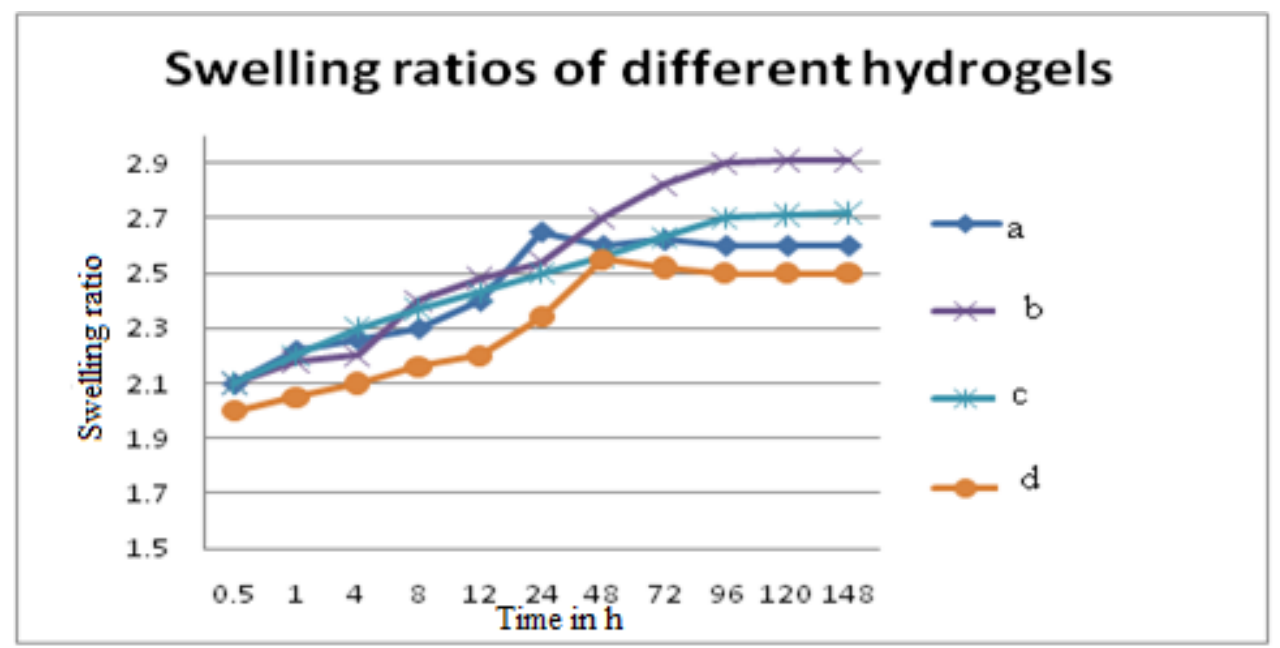

(a) Collagen, (b) Collagen- PVP with riboflavin, (c) Collagen-PVP with ascorbic acid, (d) Collagen-PVP

Figure. 3 Maximum swelling ratios of different hydrogels $(\mathrm{mg} / \mathrm{h})$

\subsection{Measurement of thermo stability}

Collagen and collagen - PVP blend membranes with a dry weight of $7-9 \mathrm{mg}$ were sealed separately in aluminium pans before thermal analyses using a differential scanning calorimeter (Pyris, 6 DSC, Perkin Elmer, Germany). Thermo scans were recorded from $5-250^{\circ} \mathrm{C}$ with the rise in $5^{\circ} \mathrm{C}$ for every 10 min with $20 \mathrm{ml} / \mathrm{min}$ of Nitrogen purge. The heat flow during the thermo scan was obtained by Pyris manager software.

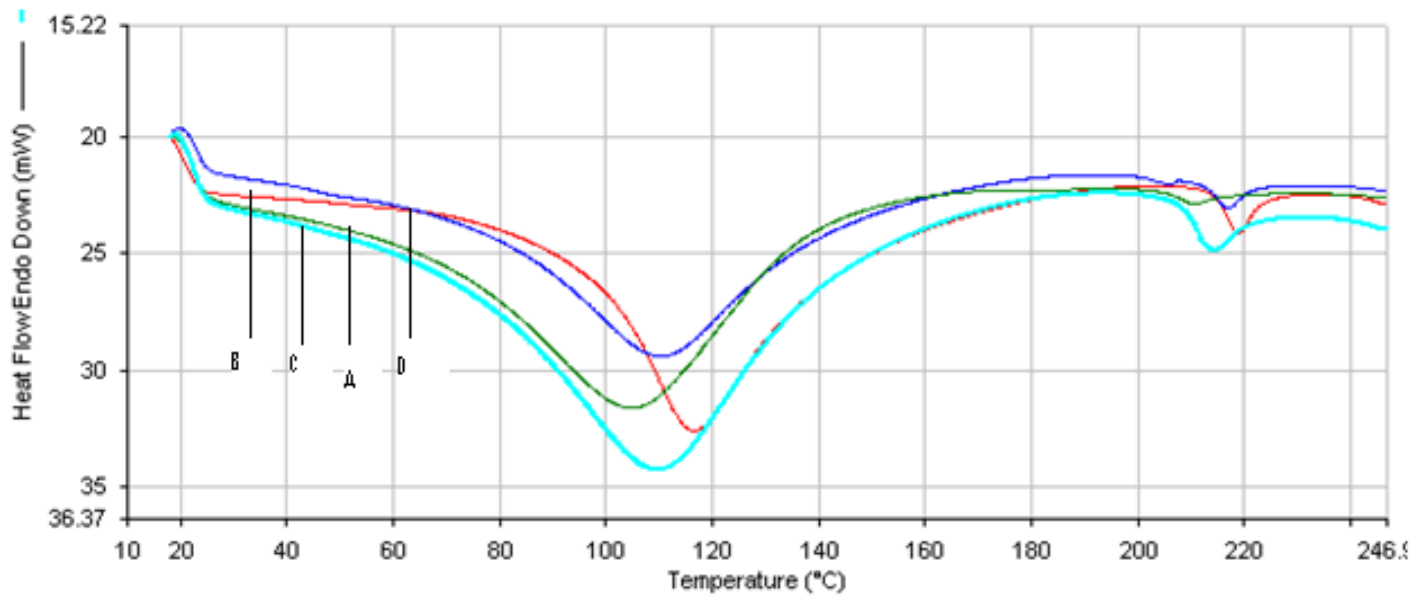

A .Non crosslinked collagen, B. collagen-PVP with riboflavin, C. Collagen-PVP with ascorbic acid, D. Collagen-PVP

Figure. 4 Thermoscans of different treatment groups of hydrogels

Thermal analysis was performed to understand the stability of hydrogels. Fig. 4 was the representatives of the thermoscans for different samples. Denaturation temperature depends upon the amount of water bonded to the collagen: the higher the water content the lower is the temperature. For the absolute dried collagen it will be nearer to $200^{\circ} \mathrm{C}$, but the common denaturation temperature for collagen is $117^{\circ} \mathrm{C}$ (Yang and Zhang, 2001) [22]. Hydrogels which were crosslinked showed two peaks: the first one which is around $118^{\circ} \mathrm{C}$ for collagen $-\mathrm{PVP}$ with riboflavin crosslinked and $115^{\circ} \mathrm{C}$ for ascorbic acid crosslinked hydrogels. The second peak was around 220 and $218^{\circ} \mathrm{C}$ respectively, which was characteristic of complete degradation of the materials. In the control groups like non crosslinked collagen and collagen -PVP blend, there was an endothermic first peak at around $114^{\circ} \mathrm{C}$ and $110^{\circ} \mathrm{C}$, second one at around 210 and $214^{\circ} \mathrm{C}$. The broad endothermic peak between $80-130^{\circ} \mathrm{C}$ indicates that these hydrogels were strongly crosslinked and required more heat for denaturation.

\subsection{Measurement of tensile testing}

The dimensions of collagen membranes were measured using a digital height gauge (Heidenhain VRZ405) and digital callipers (Mitutoyo, CD6CSX). A tensile testing machine (LLOYD LRX Tensometer, UK) connected to a load cell of $10 \mathrm{~N}$ and the collagen membranes were mounted to the custom made fixtures and a 
uniaxial force at a constant strain rate of $5 \mathrm{~mm} / \mathrm{min}$ was applied. Young's modulus for each sample was calculated from stress strain relationship.

The mechanical strength of the hydrogels was presented in Fig. 5. Young's modulus was calculated from the stress strain relationship and the hydrogels which were crosslinked with ascorbic acid were superior in their strength over collagen-PVP blends crosslinked with riboflavin. The hydrogels which were strongly crosslinked showed maximum load $(\mathrm{N})$ and high \% strain and this tendency was decreased with non crosslinked hydrogels.

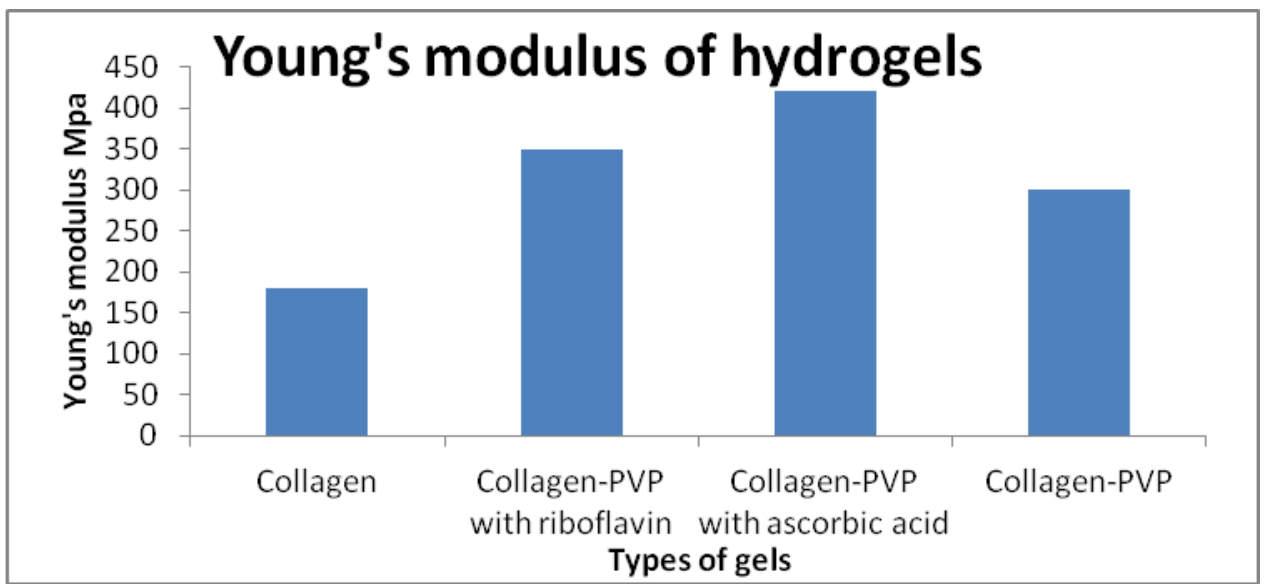

Figure. 5 Young's modulus of different hydrogels

\subsection{Protein release studies}

Bovine serum albumin $(\mathrm{mg} / \mathrm{ml})$ was dissolved in Phosphate buffered saline and it was dropped slowly on to the dried hydrogel membranes with a micro syringe until they were saturated. After that the hydrogels were air dried and weighed again using sensitive balance (Sartorius, AGB Scientifics Ltd) to get the amount of entrapped protein.

To study the release pattern of this protein, hydrogel pieces $(4-5 \mathrm{mg})$ were immersed in PBS solution and $1 \mathrm{ml}$ of the solution was taken out from each and assayed for released protein at $540 \mathrm{~nm}$ using a spectrophotometer (Perkin Elmer UV/Visible Spectroscope, Lambda 2). The amount of protein released was calculated from standard graph.

Protein release from hydrogels was studied in phosphate buffered saline $\mathrm{pH}$ 7. The release profiles of these proteins were shown in Fig. 6. Hydrogels crosslinked with riboflavin and ascorbic acid gave a controlled rate of protein release against time. They showed maximum release of $0.48 \mu \mathrm{g}$ and $0.45 \mu \mathrm{g}$ in case of riboflavin and ascorbic acid at $96 \mathrm{~h}$ of time respectively. In case of non crosslinked collagen-PVP blend hydrogels showed maximum release at $24 \mathrm{~h}(0.43-0.44 \mu \mathrm{g})$.Collagen showed maximum release $(0.42 \mu \mathrm{g})$ at $12 \mathrm{~h}$. There was a good protein release rate in case of crosslinked hydrogels because of their porosity and stability in aqueous media.

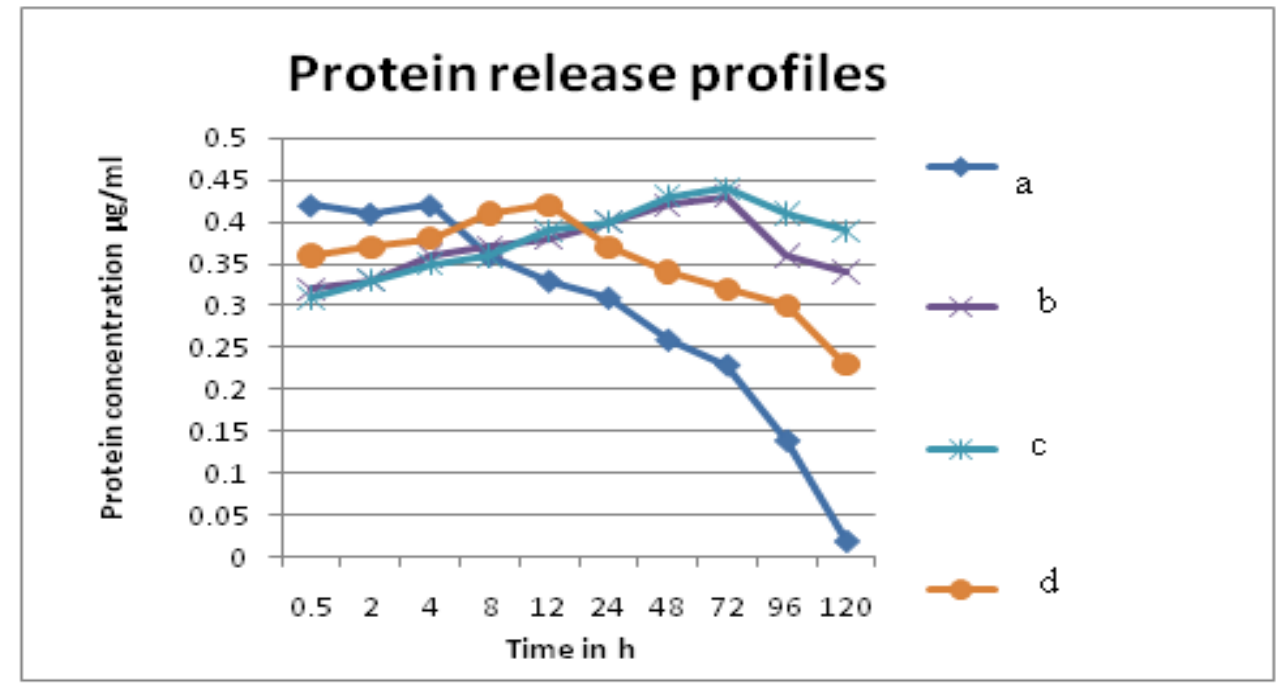


Fig. 6 Protein release profiles of different hydrogels

\section{Conclusion}

The main focus of this study was to improve the physicochemical properties of collagen based hydrogels for protein delivery by photochemical crosslinking using vitamins as photochemical initiators. This method is more efficient and less time consuming when compared to other methods. The important thing aimed in this work was the effective use of vitamins like riboflavin and ascorbic acid as photochemical sensitizers in the process of collagen crosslinking with PVP. The data obtained from the above experimental parameters indicated that these vitamins are as efficient as other chemical crosslinking agents like glutaraldehyde. Photochemically crosslinked collagen-PVP blends with the vitamins shows superiority over the control samples without any photoinitiators. Crosslinking influences the swelling property of collagen gels which is the major ascribed characteristic feature for controlled drug delivery studies. Swelling of hydrogel helps in the diffusion of proteins and other molecules from inside to outside and outside to inside in the microenvironment of the hydrogels. In the current study the gels show good swelling ratios against time.

The high Young's modulus of around 350.50 to $424.36 \mathrm{Mpa}$ for crosslinked hydrogels indicates improvement in mechanical properties which is a critical feature in the preparation of the hydrogels and this technology particularly enhanced the thermal stability of collagen membranes. Their broad endothermic peak between $80-130^{\circ} \mathrm{C}$ and their high denaturation temperatures between $200-220^{\circ} \mathrm{C}$ clearly suggests the crosslinking and strong interaction between collagen-collagen and collagen-PVP. This demonstrates that the crosslinking was caused by covalent and thermo-stable intermolecular interactions.

In addition to thermal analysis, an IR spectral shift of amide linkages also confirms the existence of intermolecular interaction of collagen molecules in hydrogels. The other interesting fact involved here is that ascorbic acid crosslinked hydrogels are competitive to the riboflavin crosslinked gels. Ascorbic acid made the hydrogels more hydrophilic and stronger, similar to the riboflavin crosslinked gels. Moreover it easily dissolves in water and there is no need for any specific solvents for dissolution. The present study and experimental results showed that the photochemical crosslinking with vitamins as photochemical initiators is able to produce hydrogels with fine porous structures and with improved physicochemical properties. These criteria are very important in the designing and manufacturing of collagen based hydrogels for controlled drug delivery studies and also as a scaffold for cell and tissue culture applications.

\section{Acknowledgements}

This study was supported in parts by grants from both Enterprise Ireland and the Athlone Institute of Technology research and development fund.

\section{References}

[1]. K.T. Nguyen, J.L. West, Photopolymerizable hydrogels for tissue engineering applications, Journal of Biomaterials.2002;23:43074314.

[2]. W.E. Hennink, C.F. van Nostrum, Novel cross linking methods to design hydrogels, Journal of Advanced Drug Delivery Reviews, 54, 2002, 13-36.

[3]. M. Ahearne, Y. Yang, K. Y. Then and K. K Liu, Non-destructive mechanical characterisation of UVA/riboflavin crosslinked collagen hydrogels, Journal of Ophthalmology,.92, 2002, 268-271.

[4]. K. Fujioka, M. Maeda, T. Hojo, A. Sano, Protein release from collagen matrices. Advanced Drug Delivery Reviews, 31 , 1998, 247266.

[5]. N. Nishad Fathima, M. Chandra Bose, J. Raghava Rao, U.N. Balachandran, Stabilization of type I collagen against collagenases (type I) and thermal degradation using iron complex. Journal of Inorganic Biochemistry, 100, 2006, 1774-1780.

[6]. A. Sionkowska, J. Kozlowska, A. Planecka, J. Skopinska-Wisniewska, Photochemical stability of poly(vinyl pyrrolidone) in the presence of collagen Polymer Degradation and Stability, 93 , 2008, 2127-2132.

[7]. S. Benamer, M. Mahlous, A. Boukrif, B. Mansouri, S. Larbi Youcef, Synthesis and characterisation of hydrogels based on poly(vinyl pyrrolidone). Nuclear Instruments and. Methods in Physics Research B 248, 2006, 284-290.

[8]. H. Kaplan , A. Guner, Characterization and determination of swelling and diffusion characteristics of poly(N-vinyl-2-pyrrolidone) hydrogels in water, Journal of Applied Polymer Science 78, 2000, 994-999.

[9]. K.P. Rao, K.T. Joseph, CRC Press, Collagen graft copolymers and their biomedical applications, in: M.E. Niimi (Ed.), Collagen Volume III Biotechnology, Boca Raton, FL. 1988; 63-86.

[10]. A. Sionkowska, Interaction of collagen and poly vinyl pyrrolidone in blends, European polymer Journal, 39, 2003,2135-2140.

[11]. C.R. Cervantes Sanehez ,E. Olaya , M. Testas , N. Garcia Lopez , G. Coste, G. Arrellin, Collagen -PVP , a collagen modulator , decreases intrperitoneal adhesions. J Surg Res. 2003; 110 (1): 207-215.

[12]. A. Sionkowska , M. Wisniewski, H. Kaczmarek, J. Skopinska , P. Chevallier, D. Mantovani, The influence of UV irradiation on surface composition of collagen/PVP blended films, Applied Surface Science, 253, 2006,1970-1976.

[13]. J. Rosiak , K.Burczak , J. Olejniczak , W. Pekala, Polimery w medycynie , Nova science (Publ) Current topics in polymer research, 1989, XIX, 3-4.

[14]. D. Balasubramanian, X .Du, J.S Zigler, The reaction of singlet oxygen with proteins, with special reference to crystallins, Journal of Photochemistry and Photobiology, 52, 1990, 761-768.

[15]. Eskins K, Proflavin-sensitized photooxidation of 3-Methyl indole to 2-Methyl-2- methoxy-1benzoxazine, Journal of Photochemistry and Photobiology, 15, 2008, $247-252$.

[16]. W. J. Ray, Photochemical Oxidation, Archives of Biochemistry and Biophysics, 33, 1951, 490-497. 
[17]. B.P.Chan,T.Y.Hui,O.C.M.Chan,K.F.So,W.Lu,K.M.C.Cheung,M.D.E.Salomatina,A.Yaroslavsky, Photochemical Cross-Linking for Collagen-Based Scaffolds: A Study on Optical Properties, Mechanical Properties, Stability, and Hematocompatibility. Journal of Tisssue Engineering, 13, 2007, 73-85.

[18]. M. Ahearne, Y. Yang, K Y Then, K-K Liu. Non-destructive mechanical characterisation of UVA/riboflavin crosslinked collagen hydrogels, Journal of Ophthalmology, 92, 2008; 268-271.

[19]. R. J. Williams and L. Pauling, Vitamin C and Collagen. http://www.vitamincfoundation.org/collagen.html, Accessed on 7-11-2008.

[20]. S. R. Sharma, R. Poddar, P. Sen, and J. T. Andrews, Effect of vitamin C on collagen biosynthesis and degree of birefringence in polarization sensitive optical coherence tomography (PS-OCT). African Journal of Biotechnology, 12, 2008, 2049-2054.

[21]. R. Makarand, H. Anandwardhan and B. Ramesh , Growth modulation of fibroblasts by chitosan- poly vinyl pyrrolidone hydrogel /Implications for wound management, J ournal of Biological Science, 25(1), 2000, 25-31.

[22]. Hongyi Yang and Qiqing Zhang, Preparation and characterization of collagen-GAGs bioactive matrices for tissue engineering, Journal of .Material Science and Technology, 17, 2001, 495-500.

[23]. E. Leikina, M. V. Mertts, N. Kuznetsova, and S. Leikin, Type I collagen is thermally unstable at body temperature, 2002 (99), 1315. www.pnas.org_cgi_doi_10.1073_pnas.032307099.

[24]. M Ozaki, Y. Adachi, Y. Iwahori, and N. Ishii, Application of fuzzy theory to writer recognition of Chinese characters, International Journal of Modelling and Simulation, 18(2), 1998, 112-116. (8) 